\title{
The BitMap dataset: an open dataset on performance assessment of diffuse optics instruments
}

Orive-Miguel, David, Lanka, Pranav, Yang, Lin, Tagliabue, Susanna, Sudakou, Aleh, et al.

David Orive-Miguel, Pranav Lanka, Lin Yang, Susanna Tagliabue, Aleh Sudakou, Saeed Samaei, Joshua Deepak Veesa, Mario Forcione, Zuzana Kovacsova, Anurag Behera, Lionel Hervé, Turgut Durduran, Adam Liebert, Piotr Sawosz, Antonio Belli, llias Tachtsidis, Alberto Dalla Mora, Jérôme Mars, Laurent Condat, Alessandro Torricelli, Hamid Dehghani, Heidrun Wabnitz, Antonio Pifferi, "The BitMap dataset: an open dataset on performance assessment of diffuse optics instruments," Proc. SPIE 11074, Diffuse Optical Spectroscopy and Imaging VII, 110741B (11 July 2019); doi: $10.1117 / 12.2526728$ 


\title{
The BitMap dataset, an open dataset on performance assessment of diffuse optics instruments
}

\author{
David Orive-Miguel ${ }^{\mathrm{a}, \mathrm{b}}$, Pranav Lanka ${ }^{\mathrm{i}}$, Lin Yang ${ }^{\mathrm{c}}$, Susanna Tagliabue ${ }^{\mathrm{d}}$, Aleh Sudakou ${ }^{\mathrm{e}}$, Saeed \\ Samaei $^{\mathrm{e}}$, Joshua Deepak Veesa ${ }^{\mathrm{f}}$, Mario Forcione ${ }^{\mathrm{g}}$, Zuzana Kovacsova ${ }^{\mathrm{h}}$, Anurag Behera ${ }^{\mathrm{i}}$, Lionel \\ Hervéa , Turgut Durduran ${ }^{\mathrm{d}}$, Adam Liebert ${ }^{\mathrm{e}}$, Piotr Sawosz ${ }^{\mathrm{e}}$, Antonio Belli ${ }^{\mathrm{g}}$, Ilias Tachtsidis ${ }^{\mathrm{h}}$, \\ Alberto Dalla Mora ${ }^{\mathrm{i}}$, Jérôme Mars ${ }^{\mathrm{b}}$, Laurent Condat ${ }^{\mathrm{b}}$, Alessandro Torricelli ${ }^{\mathrm{i}}$, Hamid \\ Dehghani $^{\mathrm{f}}$, Heidrun Wabnitz ${ }^{\mathrm{c}}$, and Antonio Pifferi ${ }^{\mathrm{i}}$ \\ ${ }^{\mathrm{a}}$ CEA, LETI, MINATEC Campus, F-38054 Grenoble, France \\ ${ }^{\mathrm{b}}$ Univ. Grenoble Alpes, CNRS, Grenoble INP, GIPSA-lab, 38000 Grenoble, France \\ ${ }^{\mathrm{c}} \mathrm{PTB}$ - Physikalisch-Technische Bundesanstalt, Berlin, Germany \\ ${ }^{\mathrm{d}}$ ICFO - The Barcelona Institute of Science and Technology, 08860 Castelldefels, Spain \\ ${ }^{\text {e}}$ Nalecz Institute of Biocybernetics and Biomedical Engineering PAS, Warsaw, Poland \\ ${ }^{\mathrm{f}}$ School of Computer Science, University of Birmingham, Birmingham, B15 2TT, United Kingdom \\ ${ }^{\mathrm{g}}$ National Institute for Health Research Surgical Reconstruction and Microbiology Research Centre, University Hospitals \\ Birmingham NHS Foundation Trust, Birmingham, United Kingdom \\ ${ }^{\mathrm{h}}$ Medical Physics \& Biomedical Engineering, UCL, London, United Kingdom \\ ${ }^{\text {i }}$ Politecnico di Milano, Dipartimento di Fisica, Piazza Leonardo da Vinci 32, 20133 Milano, Italy
}

\begin{abstract}
Open Data philosophy is becoming more popular among scientists. Open Data approach aims to transform science by making high-quality and well-documented scientific data open to everybody in order to promote collaboration and transparency. In diffuse optical and near-infrared spectroscopy community, a large measurement dataset collected with state-of-the-art instrumentation applied on well-defined phantoms is still missing. Within that context, several European labs from BitMap network ${ }^{1}$ have collected diffuse optical data on standard phantoms involving the largest set of diffuse optics instruments published until now. In this work, we present a running project on the open dataset and associated reporting tools.
\end{abstract}

Keywords: diffuse optics, near-infrared spectrocopy, open data, scientific data, HDF5

\section{INTRODUCTION}

Open Data (OD) philosophy aims to make scientific results more transparent and reusable by fully disclosing the collected experimental data. Recently, the European Commission has grabbed attention into the importance of sharing scientific data with the society and suggested to scientist to embrace OD philosophy. Although, OD approach seems simple to implement, good practices must be followed to promote reusability and transparency; OD implies not only to share the generated data but also to fully document it by describing in detail aspects such as the used instrumentation, materials or procedures. That is, detailed metadata must also be included into the dataset.

In some scientific fields like particle physics, OD approach is already well established within the community, where is some cases more than one petabyte of well-documented information is freely available. ${ }^{2}$ Nevertheless, in the diffuse optical spectroscopy and imaging communities, the OD approach is not fully developed yet. Data from experiments are scattered across the Internet, difficult to compare due to the lack of metadata or simply not available. Therefore, a repository with documented diffuse optical data following well-defined standards is still missing.

In this work, partners from BitMap network present a dataset involving the largest set of diffuse optics instruments published until now. A consistent set of phantoms has been transferred to various European laboratories and several tests have been performed using three internationally agreed protocols. The whole dataset

Send correspondence to David Orive-Miguel (david.orive-miguel@cea.fr) or Antonio Pifferi (antonio.pifferi@polimi.it).

Diffuse Optical Spectroscopy and Imaging VII, edited by Hamid Dehghani, Heidrun Wabnitz, Proc. of SPIE-OSA Vol. 11074, 110741B · C 2019 SPIE-OSA · CCC code: 1605-7422/19/\$21 · doi: 10.1117/12.2526728 
will be uploaded to an OD repository in companion with detailed metadata and documentation. A standardized data format, promoted by the Society for Functional Near Infrared Spectroscopy (SFNIRS), ${ }^{3}$ will be used that includes measurements, instrumentation technical information and metadata. Reporting tools for creating an unified reporting sheet will also be provided.

\section{METHODOLOGY}

The data contained in the database was collected at seven highly-renowned European laboratories: Politecnico di Milano (POLIMI, Italy), Physikalisch-Technische Bundesanstalt (PTB, Germany), University Hospital Birmingham (UHB, UK), University of Birmingham (UoB, UK), Instytut Biocybernetyki i Inżynierii Biomedycznej (IBIB, Poland), University College London (UCL, UK) and Institut de Ciències Fotòniques (ICFO, Spain). The same experiments with same phantoms were carried out by the same researcher in all these laboratories. All the experiments were defined by standard protocols with the set of phantoms and instrumentation described below.

\subsection{Protocols}

Optical systems performance was assessed by using three well-agreed international protocols: (1) Characterization of instrument performances such as source and detectors was done under BIP protocol. ${ }^{4}$ (2) Capabilities of instruments to measure homogeneous optical properties in turbid media was assessed using MEDPHOT protocol. ${ }^{5}$ (3) Capabilities of instruments to measure absorption inhomogenities in turbid media was characterized using NEUROPT protocol. ${ }^{6}$

\subsection{Phantoms}

A consistent set of phantoms were sequentially circulated to all laboratories. Only solid phantoms were used due to their stability, reproducibility and easy-to-use characteristics. For BIP protocol responsivity phantom ${ }^{4}$ was used. A set of 32 homogeneous solid phantoms ${ }^{5}$ that cover a wide range of scattering and absorption properties were used for MEDPHOT protocol. Finally, a solid switchable phantom ${ }^{7}$ was used for NEUROPT protocol.

\subsection{Instrumentation}

Tested systems included laboratory systems, clinical prototypes and commercial devices. Also, different technologies were used, including continuous-wave $(\mathrm{CW})$, frequency domain $(\mathrm{FD})$ and time-resolved (TR) techniques. Some of the systems were broadband or multispectral spectrometers whereas others were oximeters with just a few discrete wavelengths designed for hemodynamics monitoring. Therefore, the collected data covers a wide spectrum of state-of-the-art devices. In Table 1, the characteristics of each instrument are summarized.

\begin{tabular}{|l|l|l|l|l|l|l|}
\hline Lab & System & Technique & Application & TRL & $\lambda[\mathrm{nm}]$ & \#det \\
\hline POLIMI & clinical spectrometer & TR & spectrometer & 5 & $600-1100$ & 1 \\
\hline POLIMI & large area detector stage & TR & oximeter & 3 & 670,830 & 1 \\
\hline PTB & laboratory system & TR & spectrometer & 4 & $670,750,830$ & 2 \\
\hline UHB/UoB & NIRO 200NX & CW & oximeter & 8 & $735,810,850$ & 2 \\
\hline UHB/UoB & ISS OXIPLEX-TS & FD & oximeter & 8 & 690,830 & 4 \\
\hline UHB/UoB & ISS IMAGENT & FD & oximeter & 8 & 690,830 & 4 \\
\hline IBIB & laboratory system (spect-TR) & TR & spectrometer & $5 / 6$ & $680-868$ & 2 \\
\hline IBIB & laboratory system & TR & perfusion & 4 & 760 & 1 \\
\hline UCL & laboratory system & CW & spectrometer & 6 & $704-911$ & 8 \\
\hline ICFO & clinical system & TR & oximeter & 7 & 690,830 & 1 \\
\hline
\end{tabular}

Table 1. Systems information. \#det = number of parallel detector channels and TRL $=$ Technology Readiness Level of the system under test (e.g. TRL3 = component/subsystem, TRL4 = laboratory prototype, TRL5 = clinical prototype, TRL6 = clinical system already demonstrated in clinics, TRL8 = commercial clinical device). 


\section{DATA FORMAT STANDARD}

Collected data will be stored following a modified version of the SNIRF format ${ }^{3}$ based on Hierarchical Data Format (HDF5). HDF5 format was selected because of its flexibility and easiness to access from different operative systems and the availability of libraries for most used programming languages. SNIRF format will be slightly updated to support raw TR data and to include the instrumental response functions of instruments.

Reporting tools based on Python will also be developed to provide an unified reporting sheet for all measurements following the standards, a prototype of the reporting sheet is shown at Figure 3. These tools will be made open-source so in the future any laboratory can perform the same experiments to compare their systems performance with the measurements presented in this repository.
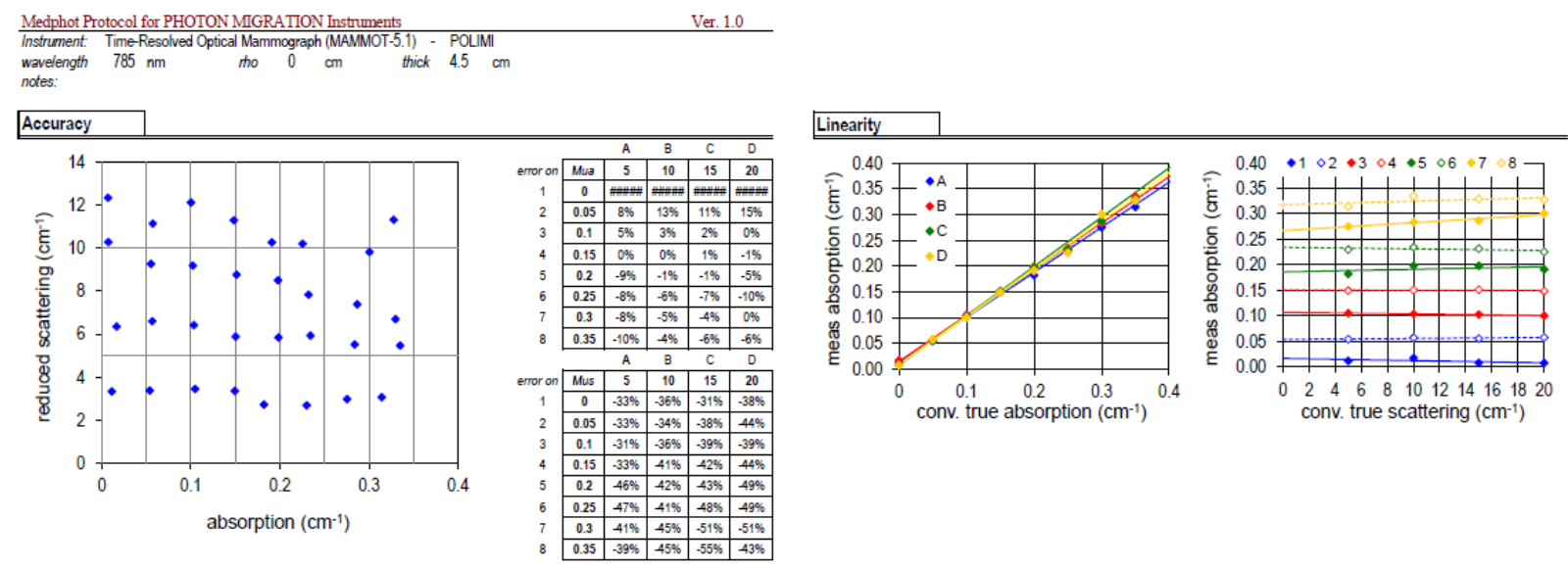

Figure 1. Example of the unified reporting sheet for the acquired data.

\section{CONCLUSIONS}

An Open Data repository is being set up with diffuse optical and near-infrared spectroscopy data collected at several European research institutes. Measurements were done following BIP, MEDPHOT and NEUROPT protocols. The data format and metadata have been well documented to promote reusability and transparency of the presented measurements.

\section{ACKNOWLEDGMENTS}

This project has received funding from the European Union's Horizon 2020 Marie Skłodowska-Curie Innovative Training Networks (ITN-ETN) programme, under grant agreement no 675332 BitMap.

\section{REFERENCES}

[1] "BitMap Initial Training Network." http://www.bitmap-itn.eu/. Accessed: 2019-01-09.

[2] "CERN Open Data Portal." http://opendata.cern.ch/. Accessed: 2019-01-09.

[3] "Shared Near Infrared File Format Specification." https://github.com/fangq/snirf/blob/master/ snirf_specification.md. Accessed: 2019-01-09.

[4] Wabnitz, H., Taubert, D. R., et al., "Performance assessment of time-domain optical brain imagers, part 1: basic instrumental performance protocol," Journal of Biomedical Optics 19, 19 - 19 - 12 (2014).

[5] Pifferi, A., Torricelli, A., et al., "Performance assessment of photon migration instruments: the medphot protocol," Appl. Opt. 44, 2104-2114 (Apr 2005).

[6] Wabnitz, H., Jelzow, A., et al., "Performance assessment of time-domain optical brain imagers, part 2: neuropt protocol," Journal of Biomedical Optics 19, 19 - 19 - 12 (2014).

[7] Pifferi, A., Torricelli, A., et al., "Mechanically switchable solid inhomogeneous phantom for performance tests in diffuse imaging and spectroscopy.," Journal of biomedical optics 20 12, 121304 (2015). 